\title{
Jervell and Lange-Nielsen syndrome with novel KCNQ1 and additional gene mutations
}

\author{
Shinichi Matsuda', Yuko Ohnuki, ${ }^{2,3}$, Mayuri Okami ${ }^{3,4,5}$, Eriko Ochiai ${ }^{6}$, Shiro Yamada (1) ${ }^{7}$, Kazumi Takahashi ${ }^{2,3}$, \\ Motoki Osawa ${ }^{6}$, Kenji Okami ${ }^{4}$, Masahiro lida ${ }^{4}$ and Hiroyuki Mochizuki ${ }^{1}$
}

\begin{abstract}
We encountered a boy with Jervell and Lange-Nielsen syndrome (JLNS) with compound heterozygous KCNQ1 mutations, maternal Trp248Phe and a novel paternal mutation, Leu347Arg. His father showed long QT (LQT) and arrhythmia. His mother was asymptomatic with no ECG abnormalities. The proband and his father had an additional mutation (SNTA1 Thr372Met), which is reportedly related to SIDS. These results suggest that multiple gene mutations influence the phenotype of KCNQ1 mutation-related arrhythmia.
\end{abstract}

Jervell and Lange-Nielsen syndrome (JLNS) (OMIM \# 220400) is a life-threatening autosomal recessive disorder characterized by a prolonged QT interval on electrocardiography (ECG) and congenital deafness ${ }^{1,2}$. Fatal arrhythmia is triggered by emotional or physical stress. JLNS is caused by homozygous or compound heterozygous mutations in either the KCNQ1 or KCNE1 gene. Without clinical intervention, the prognosis of JLNS is poor. JLNS is the most serious of the major variants of long-QT syndrome (LQTS). Nearly $90 \%$ of patients are symptomatic, and sudden death occurs in $>25 \%$ of patients despite beta-blocker therapy. In addition, JLNS patients begin to suffer from cardiac events very early in life. In the first year of life, $15 \%$ had already experienced a cardiac event, $50 \%$ had experienced a cardiac event by 3 years of age, and $90 \%$ had symptoms by 18 years of age ${ }^{3-5}$.

We experienced a case involving a 4-year-old boy with congenital hearing impairment and loss of consciousness. He was born at 36 weeks and 3 days of gestational age by cesarean section delivery due to fetal distress. There were no major complications after birth. A newborn hearing screening test suggested a bilateral hearing disorder,

\footnotetext{
Correspondence: Hiroyuki Mochizuki (mochihi@tokai-u.jp)

${ }^{1}$ Department of Pediatrics, Tokai University School of Medicine, Isehara, Japan ${ }^{2}$ Department of Medical Ethics, Tokai University School of Medicine, Isehara, Japan
}

Full list of author information is available at the end of the article which was confirmed by an otolaryngologist. His developmental milestones were delayed (head control, 8 months; turning over, 10 months; sitting, 12 months), and he was unable to stand at 1 year of age. In addition, he was diagnosed with iron deficiency anemia, and iron administration was started. No cardiac anomalies or arrhythmias were found at this time.

At 1 year and 11 months of age, a genetic test for congenital hearing loss and congenital cytomegalovirus infection was performed, and no abnormalities were suggested. At 2 years of age, cochlear implant surgery was performed. At 4 years and 1 month of age and 4 years and 8 months of age, he had two episodes of sudden poor complexion and loss of consciousness while running for a short time.

At 4 years and 11 months of age, he was running and suddenly fainted and fell onto his back and subsequently experienced convulsions for one minute; then, his consciousness gradually recovered. He was transported to our hospital. When he arrived at the emergency room, his consciousness was clear.

No abnormalities were observed on electroencephalography, head magnetic resonance imaging or magnetic resonance angiography. There was a tendency toward bradycardia for his age, with a heart rate of $\sim 60$ beats/min. The prolonged QT interval was diagnosed according to Bazett's corrected QT interval (QTcB), 


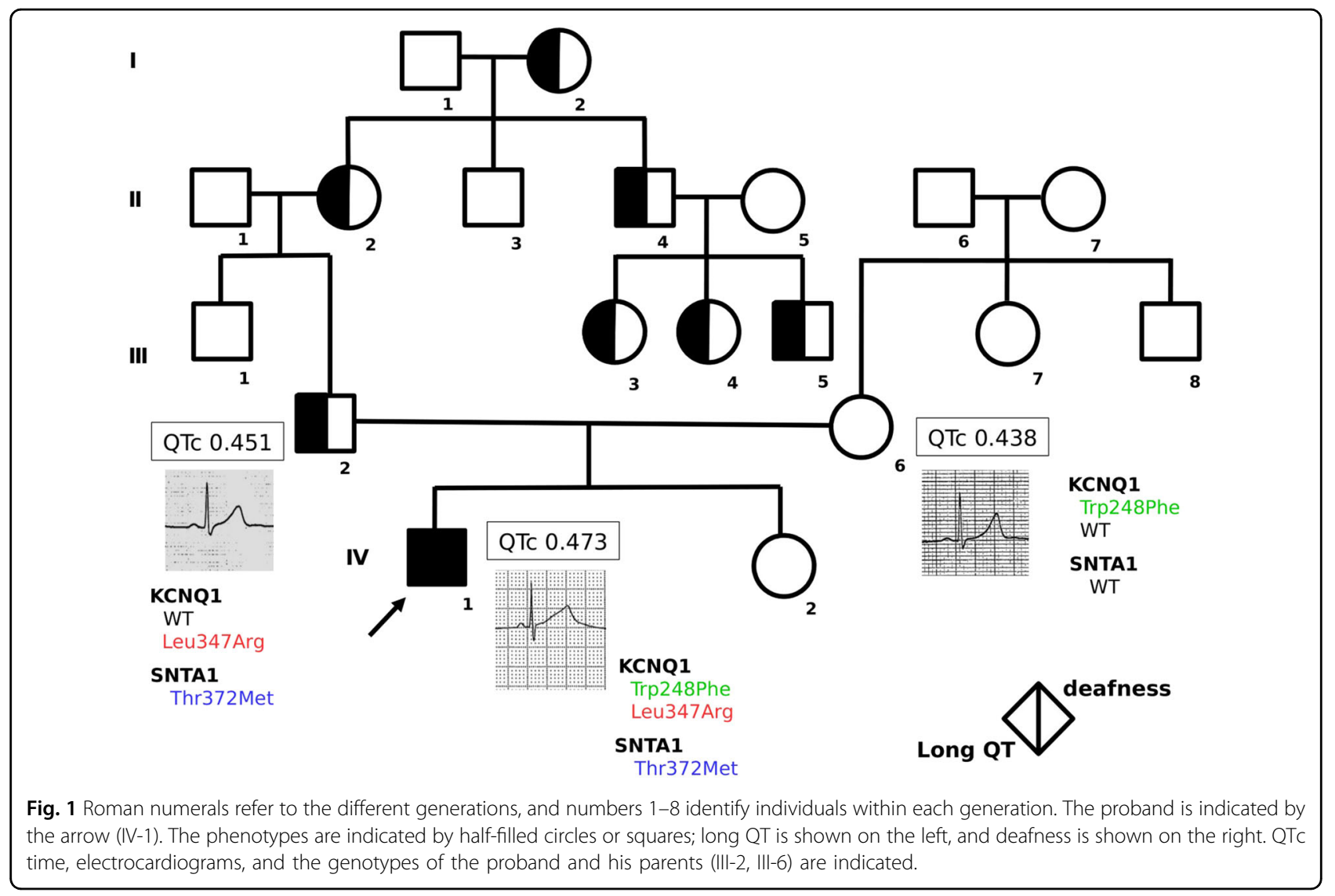

which was $470-500 \mathrm{~ms}$. A notched $\mathrm{T}$ wave was also recognized in three leads on ECG. Echocardiography revealed normal cardiac function with no cardiac malformations. No electrolyte abnormalities were detected.

The patient fulfilled nine points of the long QT (LQT) diagnostic criteria (Schwartz's score). Furthermore, his symptoms, prolonged QT combined with deafness, QTc 470-500 ms, bradycardia ( $<60$ beats $/ \mathrm{min})$, notched $\mathrm{T}$ wave in three leads, and $\mathrm{T}$ wave alternans, are high-risk factors for cardiac arrest. For the prevention of lethal arrhythmia with prolonged QT, beta-blocker treatment was initiated. Based on the genetic tests described below, he was diagnosed with prolonged QT syndrome due to the KCNQ1 (LQT1) mutation.

His exercise strength and school lifestyle were restricted according to "the management criteria for children with heart disease" by the Japanese Society of Pediatric Cardiology and Cardiac Surgery. Swimming and related activities were prohibited. In the 3 years after the diagnosis, he had no episodes of syncope.

Because familial LQT syndrome was strongly suspected, we held a multidisciplinary genetic counseling session for the family. Re-examination of the family history revealed that many paternal relatives had prolonged QT intervals.
Thus, genetic testing was recommended to his parents (Fig. 1).

Although many relatives had been diagnosed with QT prolongation, the paternal relatives had no history of syncope or sudden death, and no family history was noted among the maternal relatives.

Genetic testing was carried out for the proband and his parents after obtaining informed consent from the patient's parents. DNA was extracted using the QIAamp DNA Blood Mini Kit (Qiagen Inc., Hilden, Germany). DNA libraries were constructed using the Ion AmpliSeq $^{\mathrm{TM}}$ Library Kit 2.0 (Thermo Fisher Scientific Inc., MA, USA). Libraries were prepared according to the manufacturer's protocol. Ion AmpliSeq ${ }^{\mathrm{TM}}$ Inherited Disease Panel (Thermo Fisher Scientific Inc.) was used as the primer set. Emulsion PCR (emPCR) was performed using the Ion OneTouch 2 instrument (Thermo Fisher Scientific Inc.) with the Ion PGM Template OT2 $200 \mathrm{Kit}$ (Thermo Fisher Scientific Inc.) in accordance with the manufacturer's protocol. Sequencing was performed using the Ion $\mathrm{PGM}^{\mathrm{TM}}$ Sequencer with the Ion PGM Sequencing 200 Kit v2 (Thermo Fisher Scientific Inc.) and the Ion 316 Chip Kit v2 (Thermo Fisher Scientific Inc.). Sequencing data were analyzed using Torrent Suite 
Software v 5.0.4 (Thermo Fisher Scientific Inc., MA, USA).

The results showed that the proband (IV-1) had the (NM_000218.3) (c.743_744delGGinsTC: p.Trp248Phe and c.1040T>G: p.Leu347Arg) compound heterozygous mutation in KCNQ1. His father (III-2) had the novel mutation p.Leu347Arg in KCNQ1. The same amino acid position mutation (c.1040T>C: p.Leu347Pro) was previously reported as a cause of LQTS ${ }^{6}$. His mother (III-6) had the p.Trp248Phe mutation in KCNQ1. This variant (rs397508123) has been reported as a cause of LQT by Ohno et al. ${ }^{7}$. The same amino acid position changes (p. Trp248Arg and p.Trp248Cys) are also reported as causes of LQTS $^{8,9}$. In addition, a mutation in the $\alpha 1$-syntrophin (SNTA1) gene (NM_003098.3) c.1115C >T Thr372Met (rs1275683627), which is reported to be related to $\operatorname{SIDS}^{10}$, was recognized in the proband and his father.

These three variants are not registered in ClinVar or the 1000 Genomes database. We performed PolyPhen-2 and SIFT analyses, and the results were "probably damaging" and "damaging", respectively, for all three variants. In the gnomeAD database, the frequency of the variant SNTA1 c.1115C $>\mathrm{T}$ Thr372Met (rs1275683627) was 0.00000 (Asian) and $8.024 \times \mathrm{E}^{-6}$ (total population), and two KCNQ1 variants were not registered. The KCNQ1 variants were predicted to be likely pathogenic in the guidelines for the interpretation of sequence variants of American College of Medical Genetics ${ }^{11}$ as follows: three moderate (PM2: Absent from controls or at extremely low frequency if recessive, in the 1000 Genomes or gnomeAD databases, PM3: Detected in trans with a pathogenic variant for recessive disorders, PM5: A novel missense amino acid change occurring at the same position as another pathogenic missense change) and two supporting (PP3: multiple lines of computational evidence support a deleterious effect on the gene or gene product, SIFT: damaging, PolyPhen-2: probably damaging, and PP4: patient's phenotype or family history is highly specific for a disease with a single genetic etiology).

Until now, KCNQ1, KCNH2, and SCN5A have been reported as candidate genes for prolonged QT syndrome that may cause SIDS. CAV3, SCN3B, SCN4B, and SNTA1 are also candidate genes that may cause $\operatorname{SIDS}^{10,12}$. However, as reported by Giudicessi et al. "individuals who harbor the same LQTS-causative mutation often assume vastly different clinical courses in terms of QTc duration and frequency of cardiac events". LQTSs are not simple Mendelian-inherited disorders ${ }^{13}$.

This was the same in our case. Although the proband's father had a KCNQ1 Leu347Arg mutation and showed QT prolongation, he was asymptomatic. All of his relatives who showed QT prolongation were asymptomatic, and in deceased relatives, death was not related to cardiac events. Genetic testing was not conducted for the relatives. On the other hand, although the proband's mother had the Trp248Phe mutation in KCNQ1, which is reported to be a causative mutation of $\mathrm{JLNS}^{7}$, there was no apparent QT prolongation, and none of her relatives had QT prolongation. Symptoms only appeared in the proband, who had compound heterozygous mutations Leu347Arg and Trp248Phe in KCNQ1.

Interestingly, a mutation in SNTA1 was also detected in the proband and his father. This gene has been reported to cause SIDS, with an increase in the action potential duration (APD) or QT time, due to an increase in the steady-state component (Late-INa) of the $\mathrm{Na}+$ current in the Na channel of the heart ${ }^{10}$.

The proband and his father had mutations in both KCNQ1 and SNTA1 and showed obvious QT prolongation, whereas the father and his relatives had no history of syncope. His mother also had no history of arrhythmia or syncope. Regarding the phenotypic difference between the JLNS child and his parents, Westenskow et al. already reported that "parents who carry 1 loss-of-function mutation of KVLQT1 or KCNE1 generally have few or no symptoms"14. In our case, based on the ACMG guidelines, the responsible paternal gene mutation was considered KCNQ1. This is supported by the fact that the ECG $\mathrm{T}$ wave patterns most closely matched the KCNQ1 mutation, as previously reported ${ }^{15,16}$. However, the effect of a mutation in SNTA1 cannot be completely excluded.

According to the above results, it was once again recognized that genetic abnormalities alone cannot predict the prognosis of LQTS. Previous studies on the risk stratification of congenital LQTS ${ }^{17}$ proposed the following high-risk factors: QTc $>500 \mathrm{~ms}$, male sex, and a gene mutation in LQT1, LQT2, and LQT3. In the future, it could be possible to diagnose a patient and select an appropriate treatment according to the combination of gene mutations, not only apparently deteriorative mutations but also slightly to moderately effective mutations, and symptoms confirmed by clinicians. ECG should be repeatedly performed for patients with congenital hearing loss and repeated episodes of syncope to detect LQTS.

We herein report a new combination of genetic mutations that could cause fatal arrhythmia. Our case also suggests that while QT intervals are somewhat inherent, the additional genetic factors that lead to fatal arrhythmia may be heterogeneous.

\footnotetext{
Author details

${ }^{1}$ Department of Pediatrics, Tokai University School of Medicine, Isehara, Japan. ${ }^{2}$ Department of Medical Ethics, Tokai University School of Medicine, Isehara, Japan. ${ }^{3}$ Department of Clinical Genetics, Tokai University Hospital, Isehara, Japan. ${ }^{4}$ Department of Otolaryngology, Tokai University School of Medicine, Isehara, Japan. ${ }^{5}$ Department of Otolaryngology, Samukawa Hospital,

Samukawa, Japan. ${ }^{6}$ Department of Forensic Medicine, Tokai University School of Medicine, Isehara, Japan. ${ }^{7}$ Department of Pediatrics, Tokai University Oiso Hospital, Oiso, Japan
} 


\section{HGV database}

The relevant data from this Data Report are hosted at the Human Genome Variation Database at https://doi.org/10.6084/m9.figshare.hgv.2915 https://doi.org/10.6084/m9.figshare.hgv.2918 https://doi.org/10.6084/m9.figshare.hgv.2921

\section{Author contributions}

S.M. conceptualized the study, drafted the initial manuscript, and performed all analyses; S.M., Y.O., M.O., S.Y., and K.T. collected data and critically reviewed and revised the manuscript; E.O. and M.O. performed genetic analysis; Y.O., K.O., M.I., and H.M. commented on the analyses and critically reviewed and revised the manuscript; and all authors approved the final manuscript as submitted and agree to be accountable for all aspects of the work.

\section{Conflict of interest}

The authors declare that they have no conflict of interest.

\section{Publisher's note}

Springer Nature remains neutral with regard to jurisdictional claims in published maps and institutional affiliations.

Received: 8 December 2019 Revised: 19 August 2020 Accepted: 14 September 2020.

Published online: 15 October 2020

\section{References}

1. Jervell, A. \& Lange-Nielsen, F. Congenital deaf-mutism, functional heart disease with prolongation of the Q-T interval and sudden death. Am. Heart J. 54, 59-68 (1957).

2. Tyson, J. et al. Mutational spectrum in the cardioauditory syndrome of Jervell and Lange-Nielsen. Hum. Genet. 107, 499-503 (2000).

3. Schwartz, P. J. K. et al. The Jervell and Lange-Nielsen syndrome: natural history, molecular basis, and clinical outcome. Circulation 113, 783-790 (2006).
4. Vincent, G. M., Timothy, K. W. \& Keating, M. The spectrum of symptoms and QT intervals in carriers of the gene for the long-QT syndrome. N. Engl. J. Med. 327, 846-852 (1992).

5. Garson, A. Jr et al. The long QT syndrome in children: an international study of 287 patients. Circulation 87, 1866-1872 (1993).

6. Yuanfeng, G. et al. Common genotypes of long QT syndrome in China and the role of ECG prediction. Cardiology 133, 73-78 (2016).

7. Ohno, S., Kubota, T., Yoshida, H., Tsuji, K., Makiyama, T. \& Yamada, S. et al. A novel mutation associated with Jervell and Lange-Nielsen syndrome in a Japanese family. Circ. J. 72, 687-693 (2008).

8. Franqueza, L. et al. Long QT syndrome-associated mutations in the S4-S5 linker of KVLQT1 potassium channels modify gating and interaction with minK subunits. J. Biol. Chem. 274, 21063-21070 (1999).

9. Heidi, F. et al. Four potassium channel mutations account for $73 \%$ of the genetic spectrum underlying long-QT syndrome (LQTS) and provide evidence for a strong founder effect in Finland. Ann. Med. 36, 53-63 (2004).

10. Cheng, J. et al. Alpha1-syntrophin mutations identified in sudden infant death syndrome cause an increase in late cardiac sodium current. Circ. Arrhythm. Electrophysiol. 2, 667-676 (2009).

11. Sue, R. et al. Standards and Guidelines for the Interpretation of Sequence Variants: A Joint Consensus Recommendation of the American College of Medical Genetics and Genomics and the Association for Molecular Pathology. Genet. Med. 17, 405-424 (2015).

12. Wilders, R. Cardiac ion channelopathies and the sudden infant death syndrome. ISRN Cardiol. 2012, 846171 (2012).

13. Giudicessi, J. R. \& Ackerman, M. J. Arrhythmia risk in long QT syndrome: beyond the disease-causative mutation. Circ. Cardiovasc. Genet. 6, 313-316 (2013).

14. Westenskow, P., Splawski, l., Timothy, K. W., Keating, M. T. \& Sanguinetti, M. C. Compound mutations: a common cause of severe long-QT syndrome. Circulation 109, 1834-1841 (2004).

15. Zhang, L. et al. Spectrum of ST-T-wave patterns and repolarization parameters in congenital long-QT syndrome: ECG findings identify genotypes. Circulation 102, 2849-2855 (2000).

16. Moss, A. J. et al. ECG T-wave patterns in genetically distinct forms of the hereditary long QT syndrome. Circulation 92, 2929-2934 (1995).

17. Priori, S. G. et al. Risk stratification in the long-QT syndrome. N. Engl. J. Med. 348, 1866-1874 (2003). 